\title{
Study on Decreasing Effect of Ascorbic Acid on Algae Killing Ability of UV
}

\author{
Jing Jiang \\ Chengdu Technological University \\ Chengdu, 610000, China \\ 25859260@qq.com
}

\begin{abstract}
In this paper, the Nostoc 106 has been taken as the test algae and the antioxidant effect of ascorbic acid in the process of killing cyanobacteria by UV-C radiation has been discussed through employing the ascorbic acid as the experimental subject. At the same time, the resistance effect of ascorbic acid on UV-C in different concentrations has been also analyzed. Under normal conditions, UV-C has a strong oxidation killing ability for Nostoc 106. When the reaction temperature is $25^{\circ} \mathrm{C}$, the ultraviolet light intensity is $0.5 \mathrm{~mW} / \mathrm{cm}^{2}$ and $\mathrm{pH}$ is 6.8 . Under the condition of $30 \mathrm{~min}$ reaction time, the killing rate of test algae can reach $77 \%$ after 5 days. However, with the addition of ascorbic acid, the killing rate of Nostoc 106 can reach $35 \%$. During the experiment, the ascorbic acid has a decreased effect on UV killing power.
\end{abstract}

Keywords-chlorophyll a; ascorbic acid; Nostoc 106; UV-C

\section{INTRODUCTION}

With the great expansion of eutrophic water area, algae has increasingly become the headache problem of waterworks which takes the surface tranquil-flow water body (such as lakes) as water source and it has been also the important factor to restrict the continued growth of national economy. Due to the development of city and industry as well as the extensive use of agricultural fertilizers, a large number of urban sewage, industrial and agricultural waste water are flowing into lakes and rivers without being processed. Some nutritive salts such as nitrogen and phosphorus have been accumulated to promote that the algae and other aquatic lives are bloomed. The water clarity is declined or "water bloom" is formed. The water quality is deteriorated and odor is produced, which leads to the rapid development of eutrophication of water. For the eutrophic lakes, the algae eutrophication is obvious, such as the freshwater lake Chaohu, Xuanwu Lake of Naning and Liuhua Lake of Guangzhou $^{[1-3]}$.

As a new method to kill the algae, UV radiation has been widely applied. It can effectively damage the body cells of cyanobacteria, destroy the phycobiliprotein and degrade the chlorophyll in short times. UV-C with the wavelength of 245-280nm has a strong bactericidal ability and the ultraviolet with the wavelength of $254 \mathrm{~nm}$ has the strongest ability ${ }^{[4]}$.

However, the researches in recent years have shown that there are some defects in killing algae of UV. The killing effect is always unstable and the killing results are not always good. The algae may be appeared after a period of time. The reason is that there are some antioxidant substances in the raw water and they can resist the UV radiation so that the killing process is not completely and the killing effect is disturbed. In this paper, the ascorbic acid is taken as the experimental subject. The ascorbic acid is added during the experiment of killing cyanobacteria by UV to study the change principles of killing effect. It is found that the ascorbic acid can slow the degradation of chlorophyll, algae density and C-PC phycocyanin, weaken the killing ability of UV and play a resistance role in the killing process under the condition of ultraviolet radiation.

\section{MATERIALS AND METHODS}

\section{A. Instruments and reagents ${ }^{[5]}$}

Nostoc 106.

BG11 culture medium is employed to cultivate Nostoc sp.106.

The apparatus and instruments include: submerged UV-C device, high-speed centrifuge, spectrophotometer (722S model), ultrasonic cell grinder, UV lamp and UV radiation meter.

\section{B. Degradation experiment of ascorbic acid on chlorophyll $^{[6]}$}

$250 \mathrm{ml}$ Nostoc 106 which are grown to log phase are added into three breakers. The first one is the blank control, the second one is the UV irradiation experiment and the third one is the UV irradiation experiment with ascorbic acid $(2 \mathrm{~mm} / \mathrm{L})$. The irradiation time is $30 \mathrm{~min}$ and the light intensity is $0.5 \mathrm{~mW} / \mathrm{cm}^{2}$. It is required to employ the magnetic device for stirring and turn on the UV lamp to conduct the algae killing experiment. The experimental temperature is $25^{\circ} \mathrm{C}$ and $\mathrm{pH}$ is 6.8 . After completing the experiment, it is required to conduct the sample analysis every 24 hours.

\section{Determination of chlorophyll a $(\mathrm{Chl} a)^{[7]}$}

$7 \mathrm{~mL}$ (defined as $\mathrm{V}_{1}$ ) algae solution should be placed in $10 \mathrm{~mL}$ centrifuge tube every 24 hours and they are broken on ultrasonic cell disruptor for 30s. The working time is $1 \mathrm{~s}$ and the interval time is $2 \mathrm{~s}$. Then, it is required to add $20 \mu \mathrm{L} 1 \%$ (mass fraction) $\mathrm{MgCO}_{3}$ solution into samples and they should be centrifuged with $8000 \mathrm{r} / \mathrm{min}$ for $15 \mathrm{~min}$. The supernatant needs to be discarded. At the same time, it is necessary to add $3 \mathrm{~mL}$ acetone solution in precipitate and place them in $4^{\circ} \mathrm{C}$ refrigerator overnight. Afterwards, 18- 24h should be centrifuged with 7000 $\mathrm{r} / \mathrm{min}$ for $10 \mathrm{~min}$ and the supernatant should be placed in $7 \mathrm{~mL}$ centrifuge tube. The OD values of $750 \mathrm{~nm}, 663 \mathrm{~nm}$, 
$645 \mathrm{~nm}$ and $630 \mathrm{~nm}$ are respectively determined. The volume of supernatant in $7 \mathrm{~mL}$ centrifuge tube is $\mathrm{V}_{2}$ and the following formula is employed to calculate $\mathrm{Chl}$ $\mathrm{a}(\mathrm{mg} / \mathrm{mL})$ :

$\mathrm{Chl} \mathrm{a}=\left[11.64 \times\left(\mathrm{OD}_{663 \mathrm{~nm}}-\mathrm{OD}_{750 \mathrm{~nm}}\right)-2.16 \times\left(\mathrm{OD}_{645 \mathrm{~nm}}-\right.\right.$ $\left.\left.\mathrm{OD}_{750 \mathrm{~nm}}\right)+0.1\left(\mathrm{OD}_{630 \mathrm{~nm}}-\mathrm{OD}_{750 \mathrm{~nm}}\right)\right] \mathrm{V}_{2} / \mathrm{V}_{1}$

\section{Determination of algae density $(O D)$}

It is required to take $7 \mathrm{~mL}$ (defined as $\mathrm{V}_{1}$ ) algae solution into $10 \mathrm{~mL}$ centrifuge tube every $24 \mathrm{~h}$ and they should be broken on ultrasonic cell disruptor for $30 \mathrm{~s}$. The working time is $1 \mathrm{~s}$ and the interval time is $3 \mathrm{~s}$. The absorbance of algae solution can be measured at $750 \mathrm{~nm}$.

\section{E. Determination of C-PC phycobiliprotein content ${ }^{[8]}$}

According to Padula method, the OD values of phycobiliproteins under $620 \mathrm{~nm}$ and $650 \mathrm{~nm}$ can be measured under spectrophotometer. The calculated formula is:

$$
\mathrm{C}-\mathrm{PC}(\mathrm{mg} / \mathrm{L})=\left(166 \times \mathrm{A}_{620}\right)-\left(108 \times \mathrm{A}_{650}\right)
$$

\section{RESULTS AND DISCUSSION}

\section{A. Change principles of chlorophyll a in Nostoc 106}

In Figure 1, it is found that the degradation rate of chlorophyll a in samples under UV-C irradiation is $77 \%$ after five days under normal conditions. In the process of UV-C radiation, there are plenty of free radicals $\bullet O H$. The photosynthetic system of cyanobacteria is destroyed by $\cdot \mathrm{OH}$, which causes the passive degradation of photosynthetic pigments. The reduction of chlorophyll a can lead to the weakening of photosynthesis, thus the growth of cyanobacteria is severely limited ${ }^{[9]}$.

When the samples with ascorbic acid are irradiated by UV-C for five days, the degradation of chlorophyll a is significantly slowed and the degradation rate only reaches $35 \%$. Comparing the two samples, it is found that the ascorbic acid can make the efficiency of UV-C oxidation reduce $42 \%$, which shows the effect of antioxidant and protect the chlorophyll a in cyanobacteria. The ascorbic acid plays the removal effect on radicals ${ }^{[10]}$. After adding the ascorbic acid, the free radicals produced by UV-C radiation will firstly react with the ascorbic acid, which can reduce the oxidative damage and protect the cyanobacteria. Therefore, the activity of the samples with ascorbic acid is higher than the one of samples without ascorbic acid.

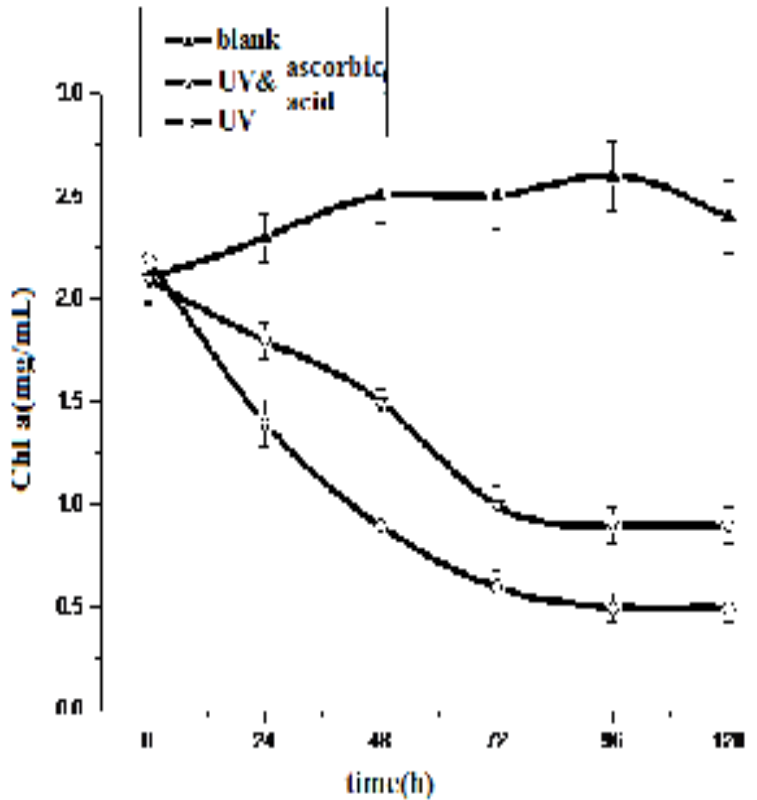

Figure 1. Change principles of chlorophyll a in Nostoc 106

\section{B. Change principles of algal biomass in Nostoc 106}

In Figure 2, the normal growth curve of cyanobacteria can be divided into the log phase and the stable phase during the experiment due that the cyanobacteria algae in logarithmic growth phase are employed in this research. The biomass of samples with UV irradiation will be significantly reduced and later they will enter the decline phase. Although the algae density of samples with ascorbic acid is reduced, it has been higher than the one of samples without ascorbic acid. They are not completely killed by ultraviolet and the total number of cells remains at a high level. The author believes that this situation is conducive to the reproduction of cyanobacteria. If the environmental conditions such as the temperature and the light which are suitable to the algae growth are satisfied, they may be greatly thrived and the rebounded growth will be appeared. Then, they will again enter the logarithmic growth phase.

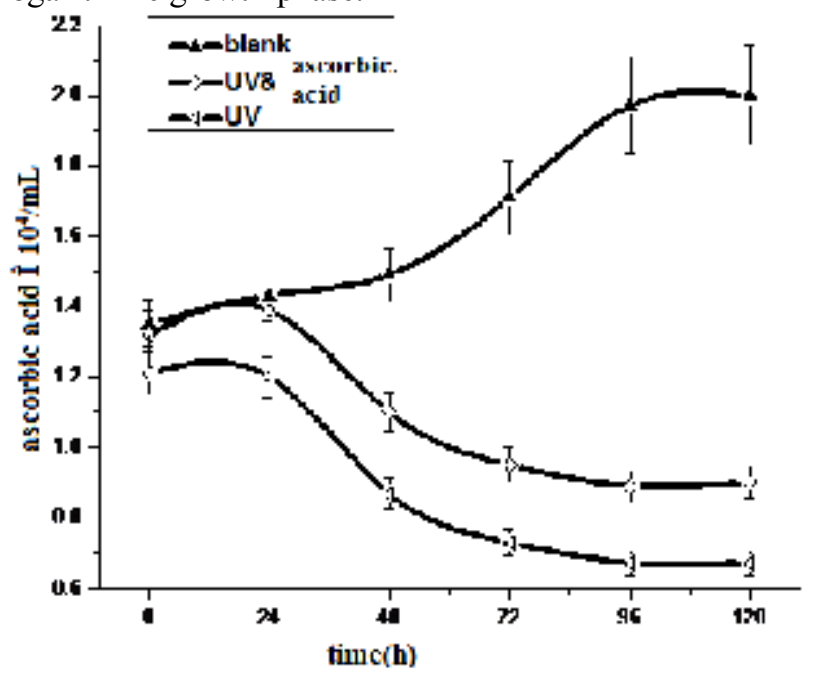

Figure 2. Change principles of algae density in Nostoc 106 


\section{Change principles of C-PC algae phycocyanin content in Nostoc 106}

In Figure 3, it is found that the C-PC content of Nostoc 106 is constantly reduced under UV radiation. The C-PC algae phycocyanin within cyanobacteria is degraded, the light-harvesting pigment system is damaged, the light energy cannot be transformed to chlorophyll, the photosynthesis in cells is inhibited and the growth of individual cells in cyanobacteria is inhibited. Then, the cyanobacteria reproduction is also inhibited. For the samples with ascorbic acid, the C-PC content is higher than the one of samples without ascorbic acid. The growth conditions of individual cells and the photosynthetic capacity are better than the ones of samples without ascorbic acid.

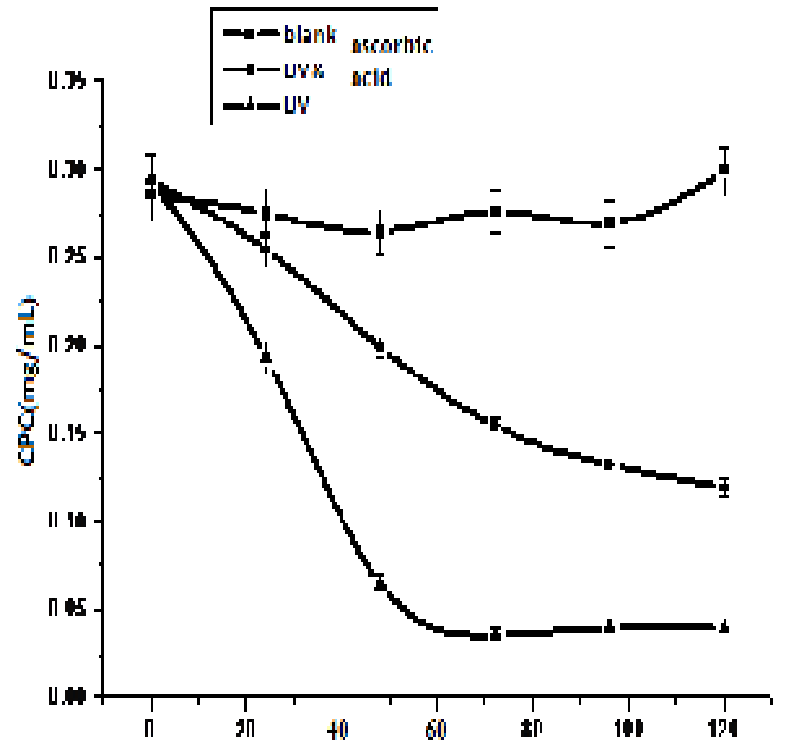

Figure 3. Change principles of algae phycocyanin in Nostoc 106

\section{Influence of ascorbic acid concentration on oxidation effect}

In Figure 4, the antioxidant effects are shown under different ascorbic acid concentrations. With the increase of ascorbic acid concentrations, the degradation rate of chlorophyll a is gradually decreased after UV-C radiation and the antioxidant effect is increased. When the concentration of ascorbic acid is $4 \mathrm{mmol} / \mathrm{L}$, the antioxidant effect will be not increased and it will be stable when the degradation rate drops to below $20 \%$. Then, it is found that there are also other killing factors in the process of ultraviolet radiation, such as the direct ultraviolet irradiation damages and the metabolic disorders caused by the destruction of cell membrane, etc.

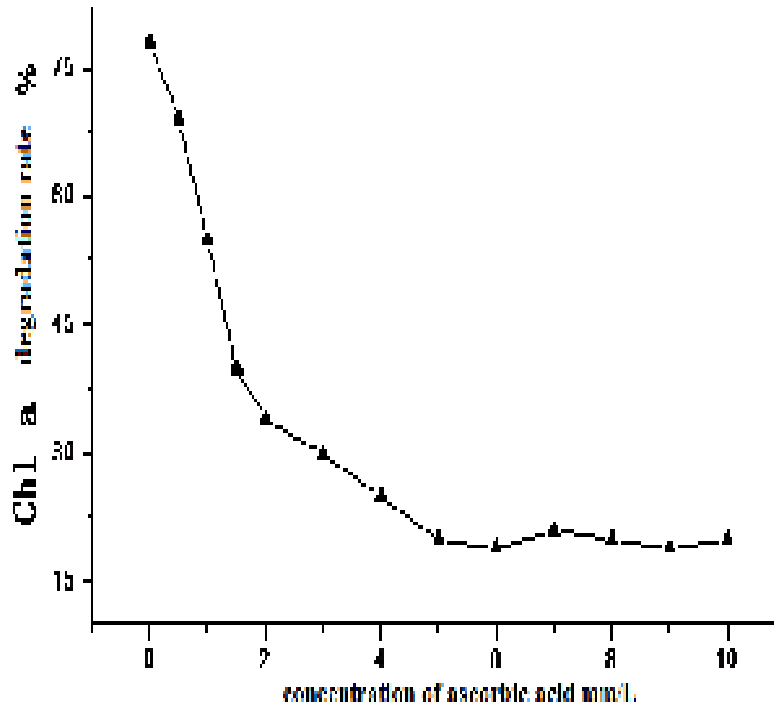

Figure 4. Influence of ascorbic acid concentration on degradation rate of chlorophyll a

\section{CONCLUSION}

In this paper, the resistance of ascorbic acid for the UV degradation effect on chlorophyll a has been analyzed. The ascorbic acid can remove the free radicals to resist the ultraviolet oxidation, slow the degradation of chlorophyll a and improve the activity of cyanobacteria. The killing rate of cyanobacteria is low in the presence of ascorbic acid, which promotes that the UV degradation effect on chlorophyll is reduced more than $40 \%$. For the algal density and the C-PC algal phycocyanin, it is also found that the ascorbic acid can decrease the UV killing effect. The UV killing is not complete, which creates conditions for the growth of cyanobacteria and the future rebound.

The results confirm that the UV-C radiation can produce the free radicals with strong oxidation and the oxidation of free radicals is the main factor in the process of algae killing. If the antioxidant substance such as ascorbic acid exists in raw water, the free radicals can be removed to reduce the killing effect. The destruction of algae photosynthesis system is limited and the degradation of chlorophyll a is slow, which can easily lead to the future rebound of cyanobacteria.

The concentration of ascorbic acid is an important factor to inhibit the ultraviolet killing effect. When the concentration reaches to $5 \mathrm{mmol} / \mathrm{L}$, the killing effect will be saturation situation and the killing effect reaches more than $50 \%$. It is found that there are also other killing factors in the process of ultraviolet radiation, such as the direct ultraviolet irradiation damages and the metabolic disorders caused by the destruction of cell membrane, etc.

In this experiment, the ultraviolet light intensity is $0.5 \mathrm{~mW} / \mathrm{cm}^{2}$, the reaction temperature is $25^{\circ} \mathrm{C}$ and $\mathrm{pH}$ is 6.8. In $30 \mathrm{~min}$ reaction time, the ascorbic acid can better weaken the UV killing power and play the antioxidant effect.

\section{ACKNOWLEDGEMENTS}

Author: Jing Jiang, born in April, 1982, han people, college teacher, instructor, master, School of Environmental Science \& Engineering, Huazhong 
University of Science and Technology. Research interests: surface water environment and control of cyanobacteria bloom. Unit: Chengdu Technological University. Address: No.1, Second part, Zhongxin Avenue, Pixian, Chengdu, Department of Electricity and technology, Chengdu Technological University. Code: 610000

\section{REFERENCES}

[1] Li Xiaolu et al. Allelopathic effects of Ceratophyllum demersum and Microcystis aeruginosa in cocultivation[J]. ACTA SCIENCE CIRCUMSTANTIAE, 2008, 11(2).

[2] Li G B, Wang G H, Song L R, et al. Lipid peroxidation in microalgae cels under simulated microgalgravity $[\mathrm{J}]$. Space medicine \& medicine engineering, 2002, 15(4): 270-272

[3] Shephard G S, Stockenstrom S, de V illiers D, et al. Degradation of microcystin toxins in a falling filmpho tocatalytic reactor with immobilized titanium dioxide catalyst [J]. Water Res, 2002, 36 (1) : $140-146$.
[4] Hu Zhangli. Biological responses of microalgae to space flight and the related molecular mechanism [D] . Ph. D. thesis of the Chinese academy of sciences, 1997.

[5] Deng Biyu et al. Improved method of measuring superoxide dismutase activity by pyrogallol autoxidation[J]. Biochemistry and Biophysics, 1991, 18(2).

[6] Parsons T R. A new method for the microdetermination of chlorophyll c in seawater[J].Mar Res, 1963, 21:164.

[7] Jing Jiang et al. Engineering Experiment and Investigation of Effect on Degradation of Cyanobacteria by the Combination of UV-C and $\mathrm{H} 2 \mathrm{O} 2$ [J].Research of Environmental Sciences, 2006, 6(19).

[8] Wang Xing et al. Effects of Di-n-butyl Phthalate (DBP) on the Growth of Cyanobacteria[J]. Journal of Wuhan University of Technology, 2006, 28(12) 48-50.

[9] M. Padula, S.Boiteux, I.Felzenszwalb, S.Menezes. Photodynamic actic of phycocyanin:damage and repair [J]. Journal of Photochemistry and Photobiology B:Biology,1996, (32):19-26.

[10] Xu Xiangrong, Wang Wenhua et al. Determination of Hydroxyl Radicals in Fenton Reaction by Colorimetric Assay and Its Application[J]. Process in Biochemistry and Biophysics, 1999, 26(1). 